\title{
Supporting research, innovation, and better outcomes for our communities
}

\author{
Chris Schneider* and Chris Bushell*
}

We're delighted to support the Journal of Community Safety and Well-Being (Journal of CSWB) in its vital work in advancing research and innovation in community safety. As a technology company, our day-to-day focus is on delivering exceptional operational efficiency and effectiveness through NicheRMS. We work every day with our police partners around world to innovate and refine the NicheRMS information management platform so it evolves with changing needs. But the work of the Journal of CSWB reminds us of the bigger picture behind the "day job" - the Journal's work to promote research and transformation will help deliver what really matters-the best possible outcomes for our communities.

As Deputy Minister, Dale McFee initiated support for the Journal, and as Editor in Chief, Community Safety Expert Norm Taylor has led its growth and success to date. Chief Dale
McFee and Norm Taylor have worked tirelessly to advance multi-agency information and data collaborations, leading towards improved social and safety outcomes for communities and individuals. We share this vision: NicheRMS advanced open platform integration capabilities are a natural fit, enabling 21st-century leaders to deliver innovative multiagency collaboration.

This is an important moment in our quest for broad and inclusive community well-being solutions. We share a collective desire for social equity and well-being for individuals, families, and communities and look forward to supporting thought leaders at the forefront of this work through our partnership.

AUTHOR AFFILIATIONS

*Niche Technology Inc., Winnipeg, MB 